Structure Determination by X-Ray Crystallography 


\title{
Structure Determination by X-Ray Crystallography Second Edition
}

\author{
M. F. C. Ladd
}

University of Surrey

Guildford, England

and

R. A. Palmer

Birkbeck College

University of London

London, England

Plenum Press - New York and London 


\section{Library of Congress Cataloging in Publication Data}

Ladd, M. F. C. (Marcus Frederick Charles).

Structure determination by $\mathrm{X}$-ray crystallography.

Includes bibliographies and index.

1. X-ray crystallography. I. Palmer, R. A. (Rex Alfred), 1936- . II. Title.

QD945.L32 1985

$548^{\prime} .83$

84-24811

ISBN 978-1-4615-7938-0 ISBN 978-1-4615-7936-6 (eBook)

DOI 10.1007/978-1-4615-7936-6

(C) 1985 Plenum Press, New York

Softcover reprint of the hardcover 1st edition 1985

A Division of Plenum Publishing Corporation

233 Spring Street, New York, N.Y. 10013

All rights reserved

No part of this book may be reproduced, stored in a retrieval system, or transmitted in any form or by any means, electronic, mechanical, photocopying, microfilming, recording, or otherwise, without written permission from the Publisher 


\section{Foreword}

X-ray crystallography provides us with the most accurate picture we can get of atomic and molecular structures in crystals. It provides a hard bedrock of structural results in chemistry and in mineralogy. In biology, where the structures are not fully crystalline, it can still provide valuable results and, indeed, the impact here has been revolutionary. It is still an immense field for young workers, and no doubt will provide yet more striking developments of a major character. It does, however, require a wide range of intellectual application, and a considerable ability in many fields.

This book will provide much help. It is a very straightforward and thorough guide to every aspect of the subject. The authors are experienced both as research workers themselves and as teachers of standing, and this is shown in their clarity of exposition. There are plenty of illustrations and worked examples to aid the student to obtain a real grasp of the subject. The practical side is encouraged by the very clarity of the theory. The examples chosen as illustrations cover the various branches of chemistry and there is useful guidance even as far as the protein field. In the later chapters dealing with the really tricky area of "getting the structure out," the treatment is well balanced, and this should help even an experienced worker to choose the most likely approach in each particular case. We seem to have passed beyond the stage at which one method is favored to the neglect of the others, and the book seems to mark a "coming of age" of $\mathrm{X}$-ray crystallography.

I wish the book the great success it undoubtedly deserves. 


\section{Preface to the Second Edition}

We have not changed the original plan and scope of this book because the first edition was, in general, well received. Additional space has been allocated to certain topics, notably experimental techniques, direct methods of phasing, and least-squares refinement. We have tried to respond to the helpful comments that we have received from reviewers and readers, and we are grateful for the interest that has been accorded to the first edition. In particular, we wish to acknowledge the kindness of Dr. C. A. Beevers for writing the Foreword to this edition.

University of Surrey

M. F. C. Ladd

Birkbeck College, London

R. A. Palmer 


\section{Preface to the First Edition}

Crystallography may be described as the science of the structure of materials, using this word in its widest sense, and its ramifications are apparent over a broad front of current scientific endeavor. It is not surprising, therefore, to find that most universities offer some aspects of crystallography in their undergraduate courses in the physical sciences. It is the principal aim of this book to present an introduction to structure determination by $\mathrm{X}$-ray crystallography that is appropriate mainly to both final-year undergraduate studies in crystallography, chemistry, and chemical physics, and introductory postgraduate work in this area of crystallography. We believe that the book will be of interest in other disciplines, such as physics, metallurgy, biochemistry, and geology, where crystallography has an important part to play.

In the space of one book, it is not possible either to cover all aspects of crystallography or to treat all the subject matter completely rigorously. In particular, certain mathematical results are assumed in order that their applications may be discussed. At the end of each chapter, a short bibliography is given, which may be used to extend the scope of the treatment given here. In addition, reference is made in the text to specific sources of information.

We have chosen not to discuss experimental methods extensively, as we consider that this aspect of crystallography is best learned through practical experience, but an attempt has been made to simulate the interpretive side of experimental crystallography in both examples and exercises.

During the preparation of this book, we have tried to keep in mind that students meeting crystallography for the first time are encountering a new discipline, and not merely extending a subject studied previously. In consequence, we have treated the geometry of crystals a little more fully than is usual at this level, for it is our experience that some of the difficulties which 
students meet in introductory crystallography lie in the unfamiliarity of its three-dimensional character.

We have limited the structure-determining techniques to the three that are used most extensively in present-day research, and we have described them in depth, particularly from a practical point of view. We hope that this treatment will indicate our belief that crystallographic methods can reasonably form part of the structural chemist's repertoire, like quantum mechanics and nmr spectroscopy.

Each chapter is provided with a set of problems, for which answers and notes are given. We recommend the reader to tackle these problems; they will provide a practical involvement which should be helpful to the understanding of the subject matter of the book. From experience in teaching this subject, the authors are aware of many of the difficulties encountered by students of crystallography, and have attempted to anticipate them in both these problems and the text. For any reader who has access to crystallographic computing facilities, the authors can supply copies of the data used to solve the structures described in Chapters 6 and 8. Certain problems have been marked with an asterisk. They are a little more difficult than the others and may be omitted at a first reading.

The Hermann-Mauguin system of symmetry notation is used in crystallography, but, unfortunately, this notation is not common to other disciplines. Consequently, we have written the Schoenflies symbols for point groups on some of the figures that depict point-group and molecular symmetry in three dimensions, in addition to the Hermann-Mauguin symbols. The Schoenflies notation is described in Appendix 3. General symbols and constants are listed in the Notation section.

We wish to acknowledge our colleague, Dr. P. F. Lindley, of Birkbeck College, London, who undertook a careful and critical reading of the manuscript and made many valuable suggestions. We acknowledge an unknown number of past students who have worked through many of the problems given in this book, to our advantage and, we hope, also to theirs. We are grateful to the various copyright holders for permission to reproduce those figures that carry appropriate acknowledgments. Finally, we thank the Plenum Publishing Company for both their interest in this book and their ready cooperation in bringing it to completion.

University of Surrey

M. F. C. Ladd

Birkbeck College, London

R. A. Palmer 


\section{Contents}

Notation . . . . . . . . . . . . . . $x i x$

\section{Chapter 1}

Crystal Geometry. I . . . . . . . . . . . . . . . . . . . 1

1.1 Introduction . . . . . . . . . . . . . . . . . . . . 1

1.2 The Crystalline State . . . . . . . . . . . . . . . 2

1.2.1 Reference Axes . . . . . . . . . . . . . . . . 5

1.2.2 Equation of a Plane . . . . . . . . . . . . . . . 9

1.2 .3 Miller Indices . . . . . . . . . . . . . . . . . . . . 10

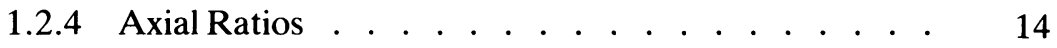

1.2.5 Zones . . . . . . . . . . . . . . . . . . . . 15

1.3 Stereographic Projection . . . . . . . . . . . . 17

1.4 External Symmetry of Crystals . . . . . . . . . . . . . 25

1.4.1 Two-Dimensional Point Groups . . . . . . . . . 25

1.4.2 Three-Dimensional Point Groups . . . . . . . . . 29

Bibliography ................ . . 48

Problems ................ . . 49

\section{Chapter 2}

Crystal Geometry.II . . . . . . . . . . . . . . . . . . . 57

2.1 Introduction . . . . . . . . . . . . . . . . 57

2.2 Lattices . . . . . . . . . . . . . . . . . . . . . . . 57

2.2.1 Two-Dimensional Lattices . . . . . . . . . . . 58

2.2.2 Choice of Unit Cell _ . . . . . . . . . . . . . . . 59

2.2.3 Three-Dimensional Lattices . . . . . . . . . . . 60 
2.3 Families of Planes and Interplanar Spacings . . . . . . . . 68

2.4 Reciprocal Lattice . . . . . . . . . . . . . . . . . . . 70

2.5 Rotational Symmetries of Lattices . . . . . . . . . . . . 74

2.6 Space Groups . . . . . . . . . . . . . . . . . . . . . 75

2.6.1 Two-Dimensional Space Groups . . . . . . . . . 77

2.6.2 Limiting Conditions Governing X-Ray Reflection . . 81

2.6.3 Three-Dimensional Space Groups . . . . . . . . 87

2.6.4 Screw Axes . . . . . . . . . . . . . . . . . . . . 90

2.6.5 Glide Planes . . . . . . . . . . . . . . . . . 94

2.6.6 Analysis of the Space-Group Symbol . . . . . . 101

Bibliography . . . . . . . . . . . . . . 102

Problems . . . . . . . . . . . . . 102

\section{Chapter 3}

Preliminary Examination of Crystals by Optical and X-Ray Methods . . . . . . . . . . . . . . . . . . 105

3.1 Introduction . . . . . . . . . . . . . . . . . 105

3.2 Polarized Light . . . . . . . . . . . . . . . . . . . . 105

3.3 Optical Classification of Crystals . . . . . . . . . . 106

3.3.1 Uniaxial Crystals . . . . . . . . . . . . . . . . 107

3.3.2 Birefringence . . . . . . . . . . . . 110

3.3.3 Biaxial Crystals . . . . . . . . . . . . . . . 112

3.3.4 Interference Figures . . . . . . . . . . . . . . 115

3.4 Direction of Scattering of X-Rays by Crystals . . . . . . . 115

3.4.1 Laue Equations for X-Ray Scattering . . . . . . . 118

3.4.2 Bragg's Treatment of X-Ray Diffraction . . . . . . 120

3.4.3 Equivalence of Laue and Bragg Treatments of X-Ray Diffraction . . . . . . . . . . . . . . . . . . 124

3.5 X-Ray Techniques . . . . . . . . . . . . . . . . . . 125

3.5.1 Laue Method . . . . . . . . . . . . . . . . . 126

3.5.2 Oscillation Method . . . . . . . . . . . . . 128

3.5.3 Ewald's Construction . . . . . . . . . . . . . . 132

3.5.4 Weissenberg Method . . . . . . . . . . . . . . 138

3.5.5 Precession Method . . . . . . . . . . . . . . . 142

3.6 Recognition of Crystal System . . . . . . . . . . . . . 145

Bibliography .. . . . . . . . . . . . . . . . . 148

Problems . . . . . . . . . . . . . . . 149 


\section{Chapter 4}

Intensity of Scattering of X-Rays by Crystals . . . . . . 153

4.1 Introduction . . . . . . . . . . . . . . . . . . 153

4.2 Path Difference . . . . . . . . . . . . . . . . 154

4.3 Mathematical Representation of a Wave: Amplitude and Phase . . . . . . . . . . . . . . . 156

4.4 Combination of Two Waves . . . . . . . . . . . . . . . 159

4.5 Argand Diagram . . . . . . . . . . . . . . . . . . . 161

4.6 Combination of $N$ Waves . . . . . . . . . . . . . . . . 162

4.7 Combined Scattering of X-Rays from the Contents of the Unit Cell ................... 164

4.7.1 Phase Difference . . . . . . . . . . . . . . . . 164

4.7.2 Scattering by Atoms . . . . . . . . . . . . . . 164

4.8 Structure Factor . . . . . . . . . . . . . . . . . . . 166

4.9 Intensity Expressions . . . . . . . . . . . . . . . . . 168

4.10 Phase Problem in Structure Analysis . . . . . . . . . . . 169

4.11 Applications of the Structure Factor Equation . . . . . . . 169

4.11.1 Friedel's Law . . . . . . . . . . . . . . . . . 169

4.11.2 Structure Factor for a Centrosymmetric Crystal . . . 171

4.11.3 Limiting Conditions and Systematic Absences . . . 173

4.11.4 Determination of Unit-Cell Type . . . . . . . . . 175

4.11.5 Structure Factors and Symmetry Elements . . . . . 176

4.11.6 Limiting Conditions from Screw-Axis Symmetry . . 179

4.11.7 Centrosymmetric Zones . . . . . . . . . . . . . 181

4.11.8 Limiting Conditions from Glide-Plane Symmetry . . 182

4.12 Preliminary Structure Analysis . . . . . . . . . . . 188

4.12.1 Practical Determination of Space Groups . . . . . . 188

Bibliography . . . . . . . . . . . . . . 192

Problems . . . . . . . . . . . . . . . . . 192

\section{Chapter 5}

Methods in X-Ray Structure Analysis. I . . . . . . . . . . 195

5.1 Introduction . . . . . . . . . . . . . . . . . 195

5.2 Analysis of the Unit-Cell Contents . . . . . . . . . . . 195

5.2.1 Papaverine Hydrochloride, $\mathrm{C}_{20} \mathrm{H}_{21} \mathrm{NO}_{4} \cdot \mathrm{HCl}$. . . . 196

5.2.2 Naphthalene, $\mathrm{C}_{10} \mathrm{H}_{8}$. . . . . . . . . . . . . . 197 
5.2.3 Molecular Symmetry . . . . . . . . . . . . . 197

5.2.4 Special Positions . . . . . . . . . . . . . . . . 198

5.2.5 Nickel Tungstate, $\mathrm{NiWO}_{4}$. . . . . . . . . . . . 199

5.3 Two Early Structure Analyses Revisited . . . . . . . . . 202

5.3.1 Sodium Chloride, $\mathrm{NaCl}$. . . . . . . . . . . . . 202

5.3.2 Pyrite, $\mathrm{FeS}_{2}$. . . . . . . . . . . . . . . 206

Bibliography . . . . . . . . . . . . . . 209

Problems ................. . 209

\section{Chapter 6}

Methods in X-Ray Structure Analysis. II . . . . . . . . . 213

6.1 Introduction . . . . . . . . . . . . . . . 213

6.2 Fourier Series . . . . . . . . . . . . . . . . . 213

6.2.1 Computation of $\psi(x)$ for a Square Wave . . . . . 214

6.2.2 Exponential Form of Fourier Expressions . . . . . 217

6.3 Representation of Crystal Structures by Fourier Series . . . 220

6.3.1 Electron Density and Structure Factors . . . . . . 220

6.3.2 Electron Density Equations . . . . . . . . . . . 223

6.3.3 Interpretation of Electron Density Distributions . . 225

6.4 Methods of Solving the Phase Problem . . . . . . . . . . 230

6.4.1 Number of Reflections in the Data Set . . . . . . . 230

6.4.2 The Patterson Function . . . . . . . . . . . . 231

6.4.3 Examples of the Use of the Patterson Function in Solving the Phase Problem . . . . . . . . . . . . 242

6.4.4 Absolute Scale of $\left|F_{o}\right|$ and Overall Temperature Factor . . . . . . . . . . . . . 257

6.4.5 Heavy-Atom Method and Partial Fourier Synthesis . $\quad 260$

6.4.6 Difference-Fourier Synthesis . . . . . . . . . . . 264

6.4.7 Limitations of the Heavy-Atom Method . . . . . . 265

6.4 .8 Isomorphous Replacement . . . . . . . . . . . . 266

6.4.9 Further Details of the Isomorphous Replacement Phasing Procedure . . . . . . . . . . . . . 274

6.4.10 Anomalous Scattering . . . . . . . . . . . 282 Bibliography . . . . . . . . . . . . . . . 286

Problems . . . . . . . . . . . . . 287 


\section{Chapter 7}

Direct Methods and Refinement . . . . . . . . 295

7.1 Introduction . . . . . . . . . . . . . . . 295

7.2 Direct Methods of Phase Determination . . . . . . . . . 295

7.2.1 Normalized Structure Factors . . . . . . . . . . 295

7.2.2 Structure Invariants and Origin-Fixing Reflections . . 298

7.2.3 Sign Determination-Centrosymmetric Crystals . . 300

7.2.4 Amplitude Symmetry and Phase Symmetry . . . . . 304

$7.2 .5 \quad \Sigma_{2}$ Listing . . . . . . . . . . . . . . . 305

7.2.6 Symbolic-Addition Procedure . . . . . . . . . . 306

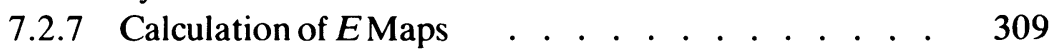

7.2.8 Phase Determination-Noncentrosymmetric Crystals 309

7.2.9 Phase Determination in Space Group $P 2_{1}$. . . . 316

7.2.10 Advantages and Disadvantages of Symbolic Addition 325

7.2.11 Multisolution Philosophy and Brief Description of the Program MUltan . . . . . . . . 326

7.2.12 Figures of Merit . . . . . . . . . . . . . . . . 328

7.2.13 Example of the Use of MULTAN . . . . . . . . . 330

7.2.14 Some Experiences . . . . . . . . . . . . . . . 331

7.3 Patterson Search Methods . . . . . . . . . . . . . . 334

7.3.1 Orientation Search . . . . . . . . . . . 335

7.3.2 Translation Search . . . . . . . . . . . . 337

7.3.3 Example of a Structure Solution by Vector Verification ............. 337

7.4 Least-Squares Refinement . . . . . . . . . . . . . . 341

7.4.1 Unit-Cell Dimensions . . . . . . . . . . . . . . 343

7.4.2 Atomic Parameters . . . . . . . . . . . . . . . 344

7.5 Molecular Geometry . . . . . . . . . . . . . . . . . 349

7.5.1 Bond Lengths and Angles . . . . . . . . . . . . 349

7.5.2 Torsion Angles . . . . . . . . . . . . . . . . 351

7.5.3 Conformational Analysis . . . . . . . . . 352

7.5.4 Mean Planes . . . . . . . . . . . . . . 355

7.6 Precision . . . . . . . . . . . . . . . 356

7.7 Correctness of a Structure Analysis . . . . . . . . . . . 357

7.8 Limitations of X-Ray Structure Analysis . . . . . . . 358

7.9 Disorder in Single Crystals . . . . . . . . . . . . . . 360

Bibliography ................. 366

Problems .............. . . 367 


\section{Chapter 8}

Examples of Crystal Structure Analysis . . . . . . . . . 371

8.1 Introduction . . . . . . . . . . . . . . 371

8.2 Crystal Structure of 2-Bromobenzo[b]indeno[1,2-e $]$ pyran (BBIP) . . . . . . . . . . . . . . 372

8.2.1 Preliminary Physical and X-Ray Measurements . . . 372

8.2.2 Intensity Measurement and Correction . . . . . . 380

8.2.3 Structure Analysis in the (010) Projection . . . . . 380

8.2.4 Three-Dimensional Structure Determination . . . . 385

8.2.5 Refinement . . . . . . . . . . . . . . . . . 389

8.2.6 Molecular Geometry . . . . . . . . . . . 391

8.3 Crystal Structure of Potassium 2-Hydroxy-3,4-dioxocyclobut1-ene-1-olate Monohydrate (KHSQ) . . . . . . . . . . 393

8.3.1 Preliminary X-Ray and Physical Measurements . . . 394

8.3.2 Intensity Measurement and Correction . . . . . . 396

8.3.3 $\Sigma_{2}$ Listing . . . . . . . . . . . . . . . . 396

8.3.4 Specifying the Origin . . . . . . . . . . . . . . 397

8.3.5 Sign Determination ． . . . . . . . . . . . . . 398

8.3.6 The EMap . . . . . . . . . . . . . . . . . . 401

8.3.7 Completion and Refinement of the Structure . . . . 403

8.4 Concluding Remarks . . . . . . . . . . . . . . . . . 405

Bibliography . . . . . . . . . . . . 406

Problems ................. 407

Appendix ... . . . . . . . . . . . . . . 411

A.1 Stereoviews and Crystal Models . . . . . . . . . 411

A1.1 Stereoviews . . . . . . . . . . . . 411

A1.2 Model of a Tetragonal Crystal . . . . . . . 414

A1.3 Stereoscopic Space-Group Drawings . . . . . . . 414

A.2 Crystallographic Point-Group Study and Recognition Scheme 414

A.3 Schoenflies' Symmetry Notation . . . . . . . . . . . . 418

A3.1 Alternating Axis of Symmetry . . . . . . . . 420

A3.2 Notation . . . . . . . . . . . . . . . 420

A.4 Generation and Properties of X-Rays . . . . . . . . 421

A4.1 X-Rays and White Radiation . . . . . . . . . . 421

A4.2 Characteristic X-Rays . . . . . . . . . . . . 422 
A4.3 Absorption of X-Rays . . . . . . . . . . . . . . 423

A4.4 Filtered Radiation . . . . . . . . . . . . . . . 424

A.5 Crystal Perfection and Intensity Measurement . . . . . 426

A5.1 Crystal Perfection . . . . . . . . . . . . . . 426

A5.2 Intensity of Reflected Beam . . . . . . . . . 428

A5.3 Intensity Measurements . . . . . . . . . . . 429

A5.3.1 Film Measurements . . . . . . . . . 430

A5.3.2 Diffractometer Geometry and Data Collection .......... . 431

A5.4 Data Processing . . . . . . . . . . . . 437

A5.4.1 Introduction . . . . . . . . . . 437

A5.4.2 Standard Deviation of Intensity . . . . 437

A5.4.3 Absorption Corrections . . . . . . . . . . 438

A5.4.4 Scaling . . . . . . . . . . . 439

A5.4.5 Merging Equivalent Reflections . . . . 440

A5.5 Synchrotron Radiation . . . . . . . . . . . . 440

A.6 Transformations . . . . . . . . . . . . . . . . . . . 441

A.7 Comments on Some Orthorhombic and Monoclinic Space

Groups . . . . . . . . . . . . . . 443

A7.1 Orthorhombic Space Groups . . . . . . . . 443

A7.2 Monoclinic Space Groups . . . . . . . . . . . 446

A.8 Vector Algebraic Relationships in Reciprocal Space . . . 446

A8.1 Introduction . . . . . . . . . . . . 446

A8.2 Reciprocal Lattice . . . . . . . . . . . . . . 446

A8.2.1 Interplanar Spacings . . . . . . . . . 448

A8.2.2 Reciprocity of Unit Cell Volumes . . . . 450

A8.2.3 Angle between Bravais Lattice Planes . . 450

A8.2.4 Reciprocity of $F$ and $I$ Unit Cells . . . . 451

A8.3 X-Ray Diffraction and the Reciprocal Lattice . . 453

A8.3.1 Bragg's Equation . . . . . . . . . 455

A8.4 Laue Photographs . . . . . . . . . . . . 457

A8.5 Crystal Setting . . . . . . . . . . . . . 458

A8.5.1 Setting Technique . . . . . . 458

A.9 Intensity Statistics . . . . . . . . . . . . . . . 460

A9.1 Weighted Reciprocal Lattice . . . . . . . . . 460

A9.1.1 Laue Symmetry . . . . . . . . . . 460

A9.1.2 Systematic Absences . . . . . . . . 460

A9.1.3 Accidental Absences .......... 461 
A9.1.4 Enhanced Averages . . . . . . . . 461

A9.1.5 Special Distributions ........ . 462

A.10 Enantiomorph Selection . . . . . . . . 465

Solutions . . . . . . . . . . . . . . . . 469

Index ................... 489 


\section{Notation}

These notes provide a key to the main symbols and constants used throughout the book. Inevitably, some symbols have more than one use. This feature arises partly from general usage in crystallography, and partly from a desire to preserve a mnemonic character in the notation wherever possible. It is our belief that, in context, no confusion will arise. Where several symbols are closely linked, they are listed together under the first member of the set.

$A^{\prime}(h k l), B^{\prime}(h k l)$. Components of the structure factor, measured along the real and imaginary axes, respectively, in the complex plane (Argand diagram)

$A(h k l), B(h k l)$. Components of the geometric structure factor, measured along the real and imaginary axes, respectively, in the complex plane

$A$. . . . . . $A$-face-centered unit cell; absorption correction factor $\AA$. . . . . . Angstrom unit; $1 \AA=10^{-8} \mathrm{~cm}=10^{-10} \mathrm{~m}$

$a, b, c \quad . \quad . \quad$ Unit-cell edges parallel to the $x, y$, and $z$ axes, respectively, of a crystal; intercepts made by the parametral plane on the $x, y$, and $z$ axes, respectively; glide planes with translational components of $a / 2, b / 2$, and $c / 2$, respectively

a,b,c . . . Unit-cell edge vectors parallel to the $x, y$, and $z$ axes, respectively

$a^{*}, b^{*}, c^{*}$. . Edges in the reciprocal unit cell associated with the $x^{*}, y^{*}$, and $z^{*}$ axes, respectively

$\mathbf{a}^{*}, \mathbf{b}^{*}, \mathbf{c}^{*}$. . . Reciprocal unit-cell vectors associated with the $x^{*}, y^{*}$, and $z^{*}$ axes, respectively

$B$. . . . . $B$-face-centered unit cell; overall isotropic temperature factor

$B_{j} \ldots \ldots$ Isotropic temperature factor for the $j$ th atom

$C$. . . . . $C$-face-centered unit cell

C . . . . . "Not constrained by symmetry to equal" 
c . . . . Velocity of light $\left(2.9979 \times 10^{-8} \mathrm{~m} \mathrm{~s}^{-1}\right)$; as a subscript: calculated, as in $\left|F_{c}\right|$

$D_{m}$. . . . . Experimentally measured crystal density

$D_{c} \quad$. . . . . Calculated crystal density

d . . . . . Interplanar spacing

$d(h k l)$. . . . Interplanar spacing of the $(h k l)$ family of planes

$d^{*}$. . . . . Distance in reciprocal space

$d^{*}(h k l) \quad$. . . Distance from the origin to the $h k l$ th reciprocal lattice point

$E, E(h k l)$. . . Normalized structure factor (centrosymmetric crystals) $\mathscr{E}(h k l)$. . . . Total energy of the $h k l$ th diffracted beam from one unit cell

e. . . . . Electron charge $\left(1.6021 \times 10^{-19} \mathrm{C}\right)$; exponential factor

$\mathbf{F}(h k l)$. . . . Structure factor for the $h k l$ th spectrum referred to one unit cell

$\mathbf{F}^{*}(h k l)$. . . Conjugate vector of $\mathbf{F}(h k l)$.

$|F|$. . . . Modulus, or amplitude, of any vector $\mathbf{F}$

$f$. . . . . Atomic scattering factor

$f_{j, \theta}, f_{j}$. . . A Atomic scattering factor for the $j$ th atom

$g$. . . . . Glide line in two-dimensional space groups

$g_{j}$. . . . . Atomic scattering factor for the $j$ th atom, in a crystal, corrected for thermal vibrations

$H$. . . . . . Hexagonal (triply primitive) unit cell

$(h k l),(h k i l)$. . $\quad$ Miller, Miller-Bravais indices associated with the $x, y$, and $z$ axes or the $x, y, u$, and $z$ axes, respectively; any single index containing two digits has a comma placed after such an index

$\{h k l\} . .$. . Form of $(h k l)$ planes

$h k l$. . . . Reciprocal lattice point corresponding to the $(h k l)$ family of planes

h . . . . . Vector with components $h, k, l$ in reciprocal space.

$h$. . . . . Planck's constant $\left(6.6256 \times 10^{-34} \mathrm{~J} \mathrm{~s}\right)$

I . . . . . Body-centered unit cell; intensity of reflection

$I(h k l) \quad$. . . . Intensity of reflection from the $(h k l)$ planes referred to one unit cell

$\mathscr{I}$. . . . . . Imaginary axis in the complex plane

i . . . . . $\sqrt{-1}$; an operator that rotates a vector in the complex plane through $90^{\circ}$ in a right-handed (anticlockwise) sense 
$J(h k l)$. . . . Integrated reflection

$K$. . . . . Reciprocal lattice constant; scale factor for $\left|F_{o}(h k l)\right|$ data

$L$. . . . . Lorentz correction factor

$M_{r}$. . . . . Relative molecular weight (mass)

$m$.... . Mirror plane

$N$. . . . . Number of atoms per unit cell

$n$ Glide plane, with translational component of $(a+$ b) $/ 2,(b+c) / 2$, or $(c+a) / 2$

$n_{1}, n_{2}, n_{3} \ldots$. Principal refractive indices in a biaxial crystal

$o$. . . . . . $\quad$ subscript: observed, as in $\left|F_{o}(h k l)\right|$

$P$. . . . . . Probability; Patterson function

$P(u, v, w)$. . Patterson function at the fractional coordinates $u, v, w$ in the unit cell

$p$. . . . . Polarization correction factor

$R$. . . . . Rhombohedral unit cell; rotation axis (of degree $R$ ); reliability factor

$\bar{R}$..... I Inversion axis

$\mathscr{R}$. . . . . Real axis in the complex plane

$R U$. . . . . Reciprocal lattice unit

$s, s(h k l), s(\mathbf{h}) \quad$. Sign of a centric reflection

$T_{j, \theta} \quad$. . . . Thermal vibration parameter for the $j$ th atom

$[U V W]$. . . Zone or direction symbol

$\langle U V W\rangle$. . . Form of zone axes or directions

u . . . . . Atomic mass unit $\left(1.66057 \times 10^{-27} \mathrm{~kg}\right)$

$(u, v, w) \quad$. . . Components of a vector in Patterson space

$\overline{U^{2}}$. . . . . Mean square amplitude of vibration

$V_{c} \quad$. . . . Volume of a unit cell

w.... . Weight factor

$x, y, z$

Spatial coordinates, in absolute measure, of a point,

$x, y, u, z$. . parallel to the $x, y,(u)$, and $z$ axes, respectively

$x, y, z \quad \ldots \quad$ Spatial fractional coordinates in a unit cell

$x_{j}, y_{j}, z_{j} \ldots$. S Spatial fractional coordinates of the $j$ th atom in a unit cell

$[x, \beta, \gamma] \quad \ldots \quad$ Line parallel to the $x$ axis and intersecting the $y$ and $z$ axes at $\beta$ and $\gamma$, respectively

$(x, y, \gamma) \quad \ldots \quad$ Plane normal to the $z$ axis and intersecting it at $\gamma$ $\pm\{x, y, z ; \ldots\} \quad . \quad x, y, z ; \bar{z}, \bar{y}, \bar{z} ; \ldots$

$Z$. . . . Number of formula-entities of weight $M_{r}$ per unit cell 
$Z_{j} \ldots . . \quad$ Atomic number of the $j$ th atom in a unit cell

$\alpha, \beta, \gamma \quad \ldots \quad$ Angles between the pairs of unit-cell edges $b c, c a$, and $\alpha^{*}, \beta^{*}, \gamma^{*} \ldots \quad$. Angles between the pairs of reciprocal unit-cell edges $b^{*} c^{*}, c^{*} a^{*}$, and $a^{*} b^{*}$, respectively

$\delta$. . . . . Path difference

$\varepsilon, \varepsilon(h k l)$. . S Statistical weight of a reflection

$\varepsilon, \omega \ldots . \quad$ Principal refractive indices for a uniaxial crystal

$\theta$.... . Bragg angle

$\lambda$..... Wavelength

$\mu$. . . . . Linear absorption coefficient

$\nu$..... . Frequency

$\nu_{n} \ldots \ldots$. Spacing between the zeroth- and $n$ th-layer lines

$\rho$. . . . . Radius of stereographic projection

$\rho(x, y, z) \quad \ldots \quad$ Electron density at the point $x, y, z$

$\Phi$. . . . . Interfacial (internormal) angle

$\phi(h k l), \phi(h), \varphi$. Phase angle associated with a structure factor

$\chi, \psi, \omega \ldots . \quad(\cos \chi, \cos \psi, \cos \omega)$ direction cosines of a line with respect to the $x, y$, and $z$ axes

$\omega$. . . . . Angular frequency

$\Omega$. . . . . Azimuthal angle in experimental methods 


\section{Structure Determination by X-Ray Crystallography \\ Second Edition}

